\title{
EXPERIMENTAL CHARACTERIZATION OF FREQUENCY DEPENDENT ELECTROSTATIC ACTUATOR FOR AQUEOUS MEDIA
}

\author{
Vikram Mukundan and Beth L. Pruitt \\ Mechanical Engineering, Stanford University \\ Stanford, CA, USA
}

\begin{abstract}
We have designed and fabricated an electrostatic actuator for operation in ionic media. The device was designed to mechanically stimulate cells and to study their mechanical properties. The frequency dependence of the device has been characterized for different media. A simple lumped capacitor circuit model for the device agrees fairly well with the experimental results. Surface treatment techniques to avoid stiction in the device have also been studied.
\end{abstract}

\section{INTRODUCTION}

Mechanical stress is known to affect cell life [1] and the role of forces acting on a cell is an important field of study. Initial work utilized soft-lithographic techniques to study the interaction between cell and substrate [2]. A number of biophysical techniques have also been developed to study the properties of cellular and sub-cellular components [3, 4]. Some of these techniques include optical tweezers, atomic force microscopy (AFM), micropipette aspiration and shear flow chambers. Optical tweezers and AFM provide high force resolution but are limited to low force ranges. Micropipette aspiration is a technique that has been successfully applied to study the properties of cell membranes and non-adherent cells. However, these techniques do not interact through the focal adhesions of a cell on a substrate. Other single cell manipulations have been demonstrated using off chip actuators. For example, Lin et al measured the forces in cardiac myocyte contraction with microfabricated polysilicon cantilevers [5]. Saif et al [6] and Yang et al [7] employed a functionalized micro-cantilever with an external piezoelectric actuator to measure the mechanical properties of fibroblasts. For on chip actuation, Chronis and Lee developed an electrothermally actuated SU-8 microgripper for cell manipulation without control of the loading profile[8]. Most similar to our design, the dual comb electromechancial nano-material testing devices developed by Zhu and Espinosa are used in air or vaccum for characterizing carbon nanotubes [9].

The device presented here will enable simultaneous stimulation and measurement of mechanical properties. We have incorporated two pairs of comb-drives to enable drive and sensing functions for force-displacement, strain rate and cyclic loading characterization of cells patterned and cultured on the devices. The actuator is designed with thin-film gold islands to be functionalized with molecules suitable for cell adhesion. Ionic shielding effects are circumvented by operating the device at high frequency. The system response is estimated from lumped model capacitor models for the electrodes, which can be related to the properties of the electrolyte using the theory of diffuse charges.

\section{THEORY}

The comb-drive actuator comprises interdigitated pairs of electrodes, with one suspended and free to move. When a voltage $V$ is applied across the electrodes, the electrostatic force is balanced by the stiffness in the suspension [10]. The force displacement relation is given by

$$
F=k x=\frac{N \varepsilon b}{d} V^{2}
$$

Where $k$ is the suspension stiffness, $\varepsilon$ is the permittivity of the medium. $N$ is the number of fingers, $d$ is the gap length and $b$ is the thickness of the device. However, this relation holds only for a dielectric medium in the gaps. The cells also need to be kept away from the regions of fringing electric fields. A FEMLAB (now COMSOL Multiphysics) model was used to estimate the field distribution in such a system. Figure 1 shows that the field is practically zero at locations more than $200 \mu \mathrm{m}$ above the right electrode. Moreover, the moving electrode will be grounded along with the substrate, so that the cell binding substrate is well shielded from the electric field effects.

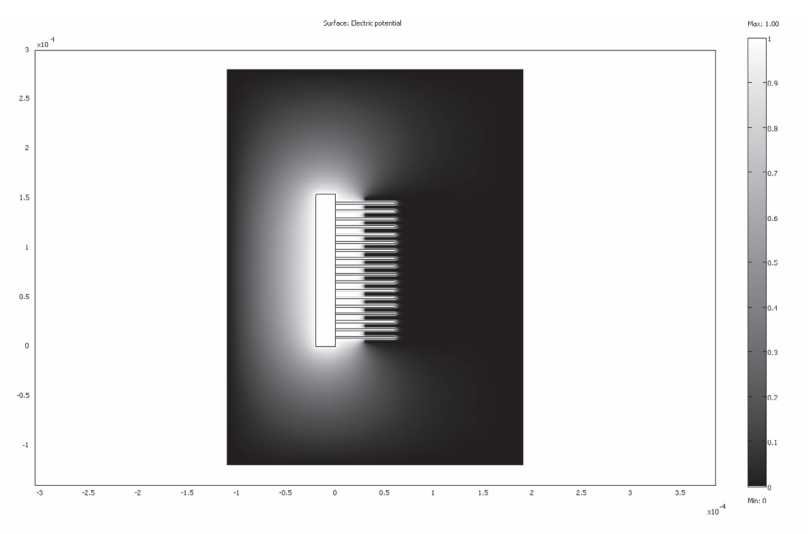

Fig 1. Electrostatic field distribution for a pair of comb-drive electrodes

Electrostatic actuation in ionic media was demonstrated by Sounart et al. [11] and Pachawagh et al [12]. They demonstrated that the ionic screening of the electrodes can be avoided by using a high frequency signal that modulates the required actuation signal. The frequency is such that the polarity of the field changes at a rate faster than the ions can respond to and the shielding of the field is avoided.

Bazant et al [13] argued that the charging dynamics of an electrode-electrolyte system is governed by the RC time constant of the system. When a solid surface is immersed in an electrolyte, 
there is spontaneous accumulation of ions near the electrodes due to surface potential at the interface. The same occurs when an external potential is applied at the electrode. The linearized expression for the double layer capacitance per unit area is given by,

$$
\mathrm{c}_{\mathrm{EDL}}=\frac{\varepsilon}{\lambda_{\mathrm{D}}}
$$

where $\lambda_{\mathrm{D}}$ is the Debye length of the double layer. Its dependence on the electrolyte properties in terms of the ionic charge valence $z$, average solute concentration $C$, Boltzmann constant $k_{b}$ and temperature $T$ is given in terms of as follows [13].

$$
\lambda_{\mathrm{D}}=\sqrt{\frac{\varepsilon \mathrm{k}_{\mathrm{b}} \mathrm{T}}{2 \mathrm{z}^{2} \mathrm{e}^{2} \mathrm{C}}}
$$

The conductivity of a symmetric electrolyte with ions of charge $z$ can be expressed as follows.

$$
\sigma=\frac{2(z e)^{2} C D}{k_{b} T}
$$

where $D$ is the diffusivity of the ions. For an electrode pair of spacing $\mathrm{d}$ immersed in a symmetric electrolyte of charge $z$, the effective time constant is as follows.

$$
\tau_{C}=\frac{\lambda_{D} d}{D}
$$

The electrode pair (Figure 2) is silicon with native oxide of thickness $\mathrm{t} \sim 2 \mathrm{~nm}$. The gap of length $\mathrm{d}$ is filled with the electrolyte medium. The circuit model for this is shown in Figure 3 where, $C_{o x}$, $C_{E D L}$ and $C_{W}$ are the capacitances of the oxide, double layer and the actuator respectively. $R_{W}$ is the resistance of the medium between the electrode, which is derived from the electrolyte conductivity relation above. The term $R_{S i}$ is not part of the electrode system, but arises due to the resistivity of the substrate through which the signal propagates. Specifically in this case, it is the resistance of the beam suspensions through which the shuttle is grounded.
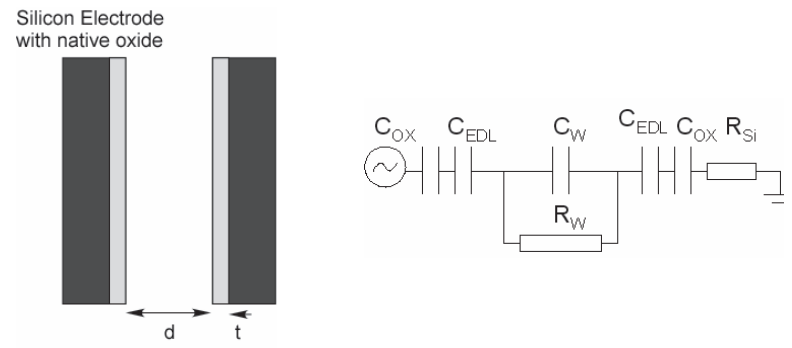

Fig 2. The electrodes are modeled as capacitors with gap $d$, and series capacitance of the dielectric native oxide.

The dominant time constant of this circuit is evaluated as follows.

$$
\tau_{\mathrm{c}}=\mathrm{RC} \mathrm{eff}=\frac{\varepsilon_{\mathrm{ox}} \varepsilon_{\mathrm{o}} d}{2 \sigma \mathrm{t}}\left[\frac{1}{1+\varepsilon_{\mathrm{ox}} \lambda \mathrm{D} / \varepsilon_{\mathrm{W}} \mathrm{t}}\right]
$$

where, $\varepsilon_{\mathrm{ox}}$ and $\varepsilon_{\mathrm{w}}$ refer to the relative permittivity of the oxide and water respectively.

\section{FABRICATION}

The devices are fabricated on a Silicon-On-Insulator (SOI) wafer; a fabrication schematic is shown in Figure 4. The silicon electrodes and suspension beams are etched into the device layer by Deep Reactive Ion Etching (DRIE) process. The metal lines comprising of Chromium/Platinum/Gold are patterned by a lift-off process. The wafer saw is used to obtain single dies before the sacrificial layer release etch. The devices are released by a timed wet-etch of the underlying oxide. The devices are then dried in a liquid $\mathrm{CO}_{2}$ critical point dryer (CPD) to avoid stiction. Figure 5 is a Scanning Electron Micrograph of a released device.
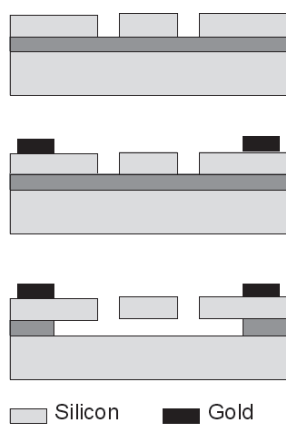

$\square$ Silicon Dioxide

Fig 4. Schematic of fabrication steps

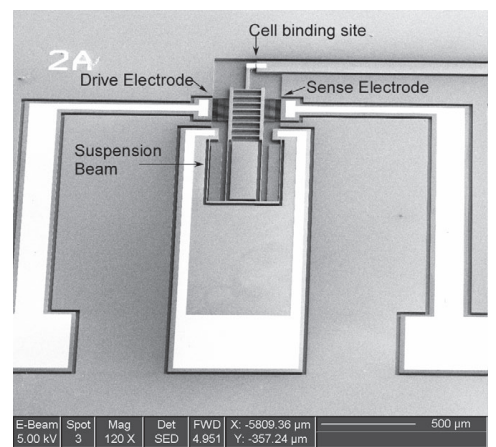

Fig 5. Scanning Electron Micrograph of a device

The device design consists of a drive electrode to actuate, a sense electrode to measure displacement by change in capacitance and a cell binding substrate with gold islands to adhere cells to. The beam suspension is designed as a folded fixed-guided beam. The stiffness of the cell and that of the beam would be in parallel, necessitating the beams to be as compliant as possible to extract stiffness properties of the cells. For fibroblasts, the average stiffness has been reported as $0.001 \mathrm{~N} / \mathrm{m}$ with a maximum elongation of about $15 \mu \mathrm{m}$ [6]. The suspension in the devices has stiffness of $k=0.24,0.58$ or $1.46 \mathrm{~N} / \mathrm{m}$. The maximum displacement of these devices is about $20 \mu \mathrm{m}$, which is set by the onset of side instability. 


\section{EXPERIMENTAL DETAILS}

The device was packaged on a ceramic Dual Inline Package (DIP) as shown in Figure 6. The actuation voltage was applied between the electrodes, with the substrate grounded. The actuator requires tens of volts for actuation in air, while it needs less than ten for actuation in water or ionic media. The DC signal was supplied through Kepco power amplifier. The AC signal was applied from an Agilent 33120A function generator. The actuator displacement was measured optically by a CCD camera attached to an upright microscope.

The package cavity was filled with the fluid for measurement. The device with the leads was setup under the microscope as shown in the Figure 7. The displacement between the two gold pads as shown in figure was measured by a code written in MATLAB. Pixel resolution of the camera enables a measurement with an accuracy of about $200 \mathrm{~nm}$ in displacement. Image processing techniques to improve resolution are under investigation as are improved signal conditioning circuits for using the sense electrodes signal for feedback control.

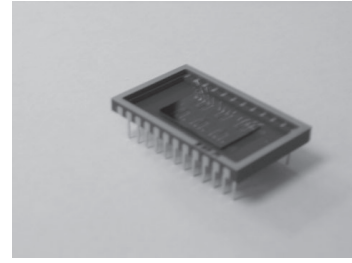

Fig 6. A chip with 6 devices is mounted and bonded in a leaded chip carrier. The carrier creates a natural well for cell culture media.

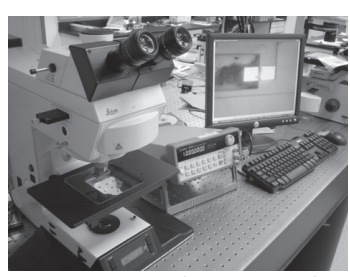

Fig 7. Experimental setup with a Leica DMRXA2 upright microscope, and Leica 350fx CCD camera.

\section{RESULTS}

\section{DISPLACEMENT CHARACTERIZATION}

Displacement as a function of frequency and amplitude of applied voltage was evaluated in air, de-ionized water, and ionic media. The displacement was found to be linear with the square of voltage. Two different tests were conducted for the same device in DI water. The first test is to measure the voltage-displacement curve and the second is to measure the frequency response of the displacement. The results are plotted in Figures 8 and 9 respectively. The theoretical estimate of the displacement is also shown.

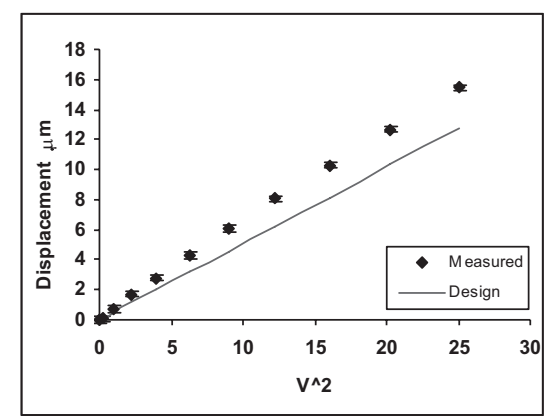

Fig 8. Actuator displacement vs. square of applied voltage for a device immersed in DI water, operated at $1 \mathrm{MHz}$.

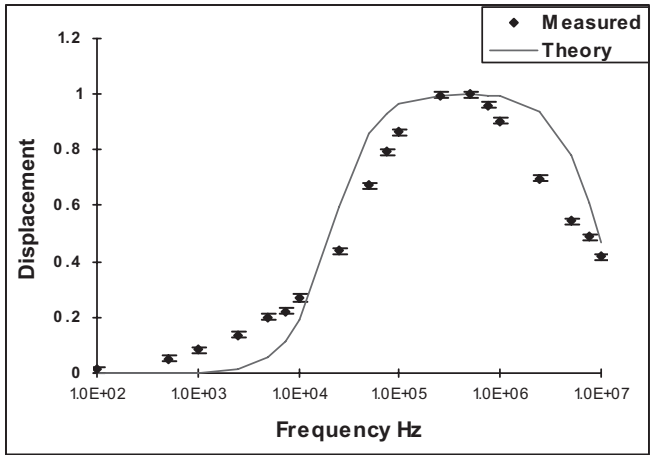

Fig 9. Actuator displacement vs. signal frequency for a constant amplitude of $3.5 \mathrm{~V}$. The plots are normalized to the maximum displacement. The model predicts the square of the voltage across the actuator for different frequencies, which is proportional to the displacement.

The frequency response does not flatten out at higher frequencies but rolls off due to substrate losses that were larger than anticipated. The path resistance due to the long suspended beams of about $0.02 \Omega-\mathrm{cm}$ is $2.67 \mathrm{k} \Omega$. This causes a low-pass effect as the resistive component dominates at higher frequencies.

In order to stimulate cells, the device needs to operate in ionic media. The device displacement was characterized in different concentrations of Potassium Chloride $(\mathrm{KCl})$ solution and Dulbecco's Modified Eagle Medium (DMEM). The results of the tests are plotted in Figures 10 and 11. The theoretical predictions according to the lumped capacitor model are also indicated on the graph.

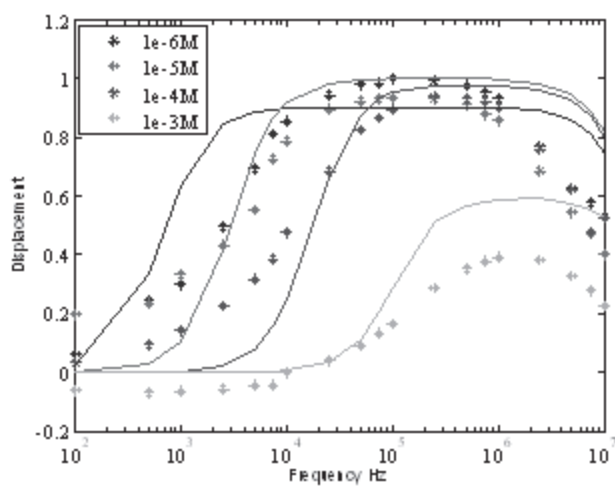

Fig 10. Normalized actuator displacement in varying concentration of $\mathrm{KCl}$ solutions. Solid lines represent theory and symbols represent data.

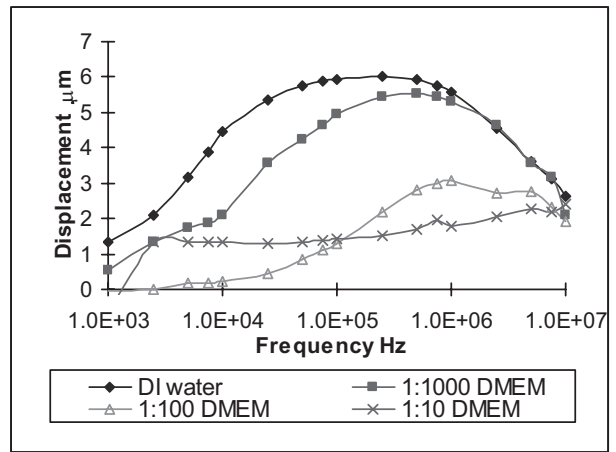

Fig 11. Actuator displacement at $5 \mathrm{~V}$ in different dilutions of DMEM, a common cell culture media. Isotonic solutions for cells require $100 \%$ DMEM. 
The curves show an increase in transition frequency with media concentration. However, there is little difference between the curves for $1 \mu \mathrm{M} \mathrm{KCl}$ and $10 \mu \mathrm{M} \mathrm{KCl}$, which is thought to be due to the presence of impurities in the solution and at the die surface. The effective conductivity of the medium is increased by these impurities. The effect of dissolved impurities would be lower in the case of higher concentrations of $\mathrm{KCl}$. It was not possible to achieve actuation for concentrations higher than $10 \mathrm{mM}$ in the case of $\mathrm{KCl}$ and less than 1:100 dilution in the case of DMEM. Applying signals of amplitudes greater than $5 \mathrm{~V}$ also caused electrolysis at the electrodes, accompanied by the release of gas bubbles.

\section{SURFACE TREATMENT}

The devices need to be released in a CPD to avoid stiction of the suspended structures. This limits the reusability of the devices as they cannot be dried and cleaned before reuse. This becomes important in the case of biological samples, where the substrates need to be cleaned between subsequent experiments. Anti-stiction self-assembled monolayer (SAM) coatings for MEMS may improve the reliability of these devices [14]. We coated the devices with 1, 1, 2, 2-Perfluorodecyltrichlorosilane (FDTS) in water vapor plasma. The contact angle after treatment was measured to be $104 \pm 3^{0}$. However, the increased surface energy made it difficult to displace air from the narrow gaps in the devices as shown in figure 12. Moreover, the highly hydrophobic surfaces likely collapsed when immersed in water as no actuation was observed in these devices. When the devices were dried and tested in air, they did not show any actuation. Further work is required to tune the surface energy to an optimum level.

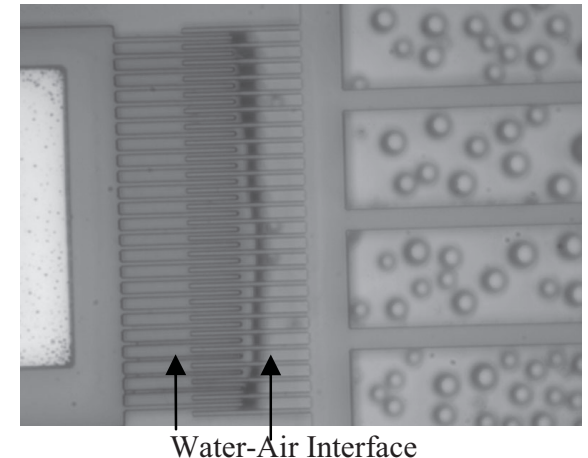

Fig 12. Image of a FDTS coated device immersed in water

\section{CONCLUSIONS}

We have designed and fabricated an electrostatic actuator for stimulating cells in ionic media. The displacement characteristics of the device have been measured in media of different concentrations. Two limitations will be addressed in the future designs. First is the potential drop across the path resistance at higher frequency. High path resistance prevents the use of the device at frequencies required by cell culture media. It will be reduced by using a higher doped substrate or by plating the conducting path with metal lines. The second limitation of these devices is their re-usability. Once immersed in media, the devices cannot be dried in air without stiction problems nor returned to the CPD in a clean room. In order to mitigate this, silane surface treatment was investigated. However, the highly hydrophobic surface causes stiction when immersed in water. Moreover the surface tension causes air bubbles to be trapped between narrow gaps in the device. Tuning the surface energy to an intermediate value may solve both the problems.

\section{ACKNOWLEDGEMENTS}

VM is supported by the Stanford Graduate Fellowship (200306). Fabrication work was performed in part at the Stanford Nanofabrication Facility (a member of the National Nanotechnology Infrastructure Network) which is supported by the National Science Foundation under Grant ECS-9731293, its lab members, and the industrial members of the Stanford Center for Integrated Systems. This work was supported by NSF CAREER Award ECS-0449400. The authors would like to thank Prof. Roya Maboudian and Brian Bush for their discussions and help with the surface treatment experiments. Travel support was generously provided by the Transducers Research Foundation.

\section{REFERENCES}

1. J. L. Tan, J.Tien, D. M. Pirone, D. S. Gray, K. Bhadriraju, C. S. Chen, "Cells Lying on a bed of microneedles: An approach to isolate mechanical forces". Proceedings of the National Academy of Sciences, 2003. 100(4): p. 1484-1489.

2. A. D. Bershadsky, N. Q. Balaban., B. Geiger, "Adhesion Dependent cell Mechanosensitivity". Annual REview of Cell Development, 2003. 19: p. 677-695.

3. K. J. Van Vliet, G.Bao., S. Suresh, "The biomechanics toolbox: experimental approaches for living cells and biomolecules". Acta Materalia, 2003. 51: p. 5881-5905.

4. G. Bao, S.Suresh., "Cell and molecular mechanics of biological materials". Nature Materials, 2003. 2: p. 715-725.

5. G. Lin, K. S. J. Pister, K. P. Roos, "Micromachined Polysilicon Heart Cell force Transducer". Journal of Microelectromechanical Sytems, 2000. 9(1): p. 9-17.

6. M. T. A. Saif, C. R. Sager., S. Coyer, "Functionalized BioMicroelectromechanical Systems Sensors for Force Response study at Local Adhesion sites of Single livling cells on substrates". Annals of Biomedical Engineering, 2003. 31: p. 950-961.

7. S. Yang, T.Saif., "Reversible and repeatable linear local cell force response under large stretches". Experimental Cell Research, 2004. 305: p. 42-50.

8. N. Chronis, L. P. Lee, "Electrothermally activated SU-8 microgripper for cell manipulation in solution", Journal of Microelectromechanical Systems, 2005, 14(4): p857-863

9. Y. Zhu, H. D. Espinosa., "An electromechanical material testing system for in situ electron microscopy and applications". Proceedings of the National Academy of Sciences, 2005. 102(41): p. 14503-14508.

10. W. C. Tang, M. G. Lim, R. T. Howe, "Electrostatic Comb Drive Levitation and Control Method"., Journal of Microelectromechanical Sytems, 1992. 1(4).

11. T. L. Sounart, T. A. Michalske., K. R. Zavadil, "FrequencyDependent Electrostatic Actuation in Microfluidic MEMS". Journal of Microelectromechanical Sytems, 2005. 14(1): p. 125-133.

12. H. V. Panchawagh, D. Serrel, D. S. Finch, T. Oreskovic, and R. L. Mahajan. "Design and Characterization of a BioMEMS device for invitro mechanical simulation of single adherent cells". in ASME International Mechanical Engineering Congress and Expedition. 2005. Orlando.

13. M. Z. Bazant, K.Thornton, Armand Ajdari, "Diffuse-charge Dynamics in Electrochemical Systems". Physical Review E, 2004. 70: p. 021506-1-23.

14. R. Maboudian, C. Carraro, "Surface Chemistry and Tribology of $M E M S^{\prime \prime}$, . Annual Review of physical Chemisty, 2004. 55: p. 3554. 